\title{
Hand Motion Analysis for Recognition of Qualified and Unqualified Welders using 9-DOF IMU Sensors and Support Vector Machine (SVM) Approach
}

\author{
Triwilaswandio Wuruk Pribadi ${ }^{1,2^{*}}$, Takeshi Shinoda ${ }^{3}$ \\ ${ }^{1}$ Department of Naval Architecture, Institut Teknologi Sepuluh Nopember, Jl. Raya ITS, Keputih, Kec. Sukolilo, \\ Surabaya 60111, Indonesia \\ ${ }^{2}$ Graduate School of Engineering, Kyushu University, 744 Motooka Nishi-ku Fukuoka 819-0395, Japan \\ ${ }^{3}$ Department of Marine Systems Engineering, Faculty of Engineering, Kyushu University, 744 Motooka Nishi-ku \\ Fukuoka 819-0395, Japan
}

\begin{abstract}
This research aimed to find out how to identify qualified and unqualified welders of shielded metal arc welding (SMAW) in the shipyard industry. A cost-effective system that can identify the welder skills in real time is needed to reduce the cost of inspection and to maintain weldment quality. In this study, 9-degree of freedom (DOF) sensors of the inertial measurement unit (IMU) were applied to measure and to record the typical hand motions of welders. These sensors consisted of an accelerometer, a gyroscope, and a magnetometer installed in a microcontroller board, known as a wearable device. The wearable device was fitted on a welder's hand to monitor and to record wrist-hand motions of both qualified and unqualified welders. The data on inertial measurements of the welder's hand motions were sent through a Bluetooth connection and then saved in a memory card of a smartphone. Some properties, such as the root mean square (RMS), correlation index, spectral peaks, and spectral power, were extracted from the time-series data to characterize hand motions. The support vector machine (SVM) method, a part of the artificial intelligence (AI) technique, was applied to classify and to recognize the typical hand motions of the two types of welders using a supervised learning approach. The validation results showed that the proposed system was able to identify qualified and unqualified welders.
\end{abstract}

Keywords: 9-DOF IMU sensors; Support vector machine; Qualified and unqualified welders

\section{Introduction}

Shielded metal arc welding (SMAW) is one type of the welding process that is commonly used in steel construction, including that in the shipbuilding industry. Such a welding process is executed mostly using the E6013 electrode, which is easily found on the market (Purnama et al., 2020). This process is still performed manually, and the weldment quality depends largely on welder skills when maintaining welding parameters, such as travel speed, the distance between electrode tips and the base metal, electric current, and voltage, with reference to the qualified welding procedure specification (WPS). The effect 
of those parameters on temperature distribution was simulated numerically using the finite are affected accordingly. Presently, such effects of hand motion in manual welding have been reduced by using automatic welding equipment and applying robotic technology as described by Sunar et al. (2019). However, the application of robotics technology in the shipbuilding industry is still limited due to many variations of the construction joint.

The verification of qualified (Q) or unqualified (UQ) welders of SMAW during the welding process is one of the problems in the shipbuilding industry. Even Q welders who stop doing welding for a certain period may experience a decline in their competency. Additionally, there are still many practices to replace Q welders with UQ ones for the purpose of cost reduction. Another case is the decrease in weldment quality when a welder applies improper hand motions due to fatigue, less focus, or different WPS applications. To overcome these problems, welding training or familiarization according to the WPS is frequently required from all welders before they are assigned to a new construction.

It has been observed and identified that the typical characteristics and steadiness of a welder's hand motions during the welding process significantly contribute to the weldment quality (Pribadi and Shinoda, 2020). If these hand motion characteristics can be monitored and recorded, the weldment quality can then be evaluated accordingly by analyzing the created graph. It is made possible using wearable sensors that can measure the linear acceleration, degree acceleration, and magnetic force of wrist-hand motions. In this case, the pattern of magnetic force signals can reflect the stability of the electric voltage and current that are applied during the welding process.

Many researchers have tried the application of motion sensors to monitor and to record various types of motion. An accelerometer sensor was used to measure the moment of the object position, including the classification of activities (Godfrey et al., 2008; Bayat et al., 2014). Recently, it was shown that the welder's hand motion pattern can be monitored and recorded by a wearable device with a 9-degree of freedom (DOF) inertial measurement unit (IMU). A multilayer perceptron approach was developed to classify welder activities in various butt-welding positions (flat-horizontal-vertical) commonly done in the shipbuilding industry (Pribadi and Shinoda, 2020). The application of a motion analysis system (MAS) is becoming increasingly popular in assisting with real-time monitoring in civil construction projects. A MAS makes a significant contribution to the manufacturing process related to increasing productivity and enhancing ergonomic aspects (Bortolini et al., 2018). Recently, sensor systems with a wearable device have been developed and researched for use by construction workers (Valero et al., 2017).

Many classifying methods that are parts of artificial intelligence (AI) technology can make the system work. The support vector machine (SVM) model is a frequently used approach to classifying activities (Gyllensten, 2010; Attal et al., 2015). The others are the multilayer perceptron model (Gyllensten, 2010; Bayat et al., 2014) and the deep convolutional neural networks model (Jiang and Yin, 2015). This utilization of AI techniques has also been combined with cloud infrastructure development (Keshavarzian et al., 2019).

\subsection{Human Motion}

Human motion or movement is described in general as related to mobility, function, and occupation, which is an end product of an intentional or unintentional consequence of joint motion and muscle activity (Everett and Kell, 2010). On one hand, anatomical and physiological functions are the focus of studies in kinesiology. On the other hand, mechanical knowledge and methods focus on biomechanical studies (Godfrey et al., 2008). Kinematics and kinetics are part of biomechanical motion. Kinematic motion focuses on the motion characteristics that do not take into account the force factor that causes the motion. 
Information regarding the position, velocity, acceleration, and both linear and angular motions can be used in kinematic analysis (Godfrey et al., 2008). Several researchers have developed the study of motions to monitor the characteristics of physiological and physical effects of the human body. Some examples are monitoring physiological conditions and activities in the construction work environment (Lee et al., 2017), measuring physical intensity (Kong et al., 2018), and measuring body fatigue of workers (Bowen et al., 2019). The use of kinematic sensors to monitor the motion characteristics is the focus of this research.

\subsection{Welder Motions in Manual Welding}

SMAW is one of the popular types of welding technology in which the process is fully controlled by the welder's hand. Welders perform welding functions and must be capable of controlling their hand movements (Erasmus+, 2014). Personal skill is essential for obtaining welding results, following applicable standards (Moore and Booth, 2015). Welders must be able to maintain arc distance and electrodes while manipulating the electrodes continuously during welding. They must undergo welding training to develop sufficient skills in electrode manipulation in various positions of butt welding, such as $1 \mathrm{G}$ flat, 2G-horizontal, and 3G-vertical (Erasmus+, 2014).

Various hand motions, such as weaving, pushing, and dragging produce the different forms of welds. A specific weaving technique should be applied to ensure sound beads of welding. Pulling techniques are utilized for maximum penetration and for generating solidlooking welds. A combination of pushing and weaving techniques is applicable in 3Gvertical welding positions to prevent the molten metal from slipping. The semi-circle weave method can be applied for keeping the overheating in the weld pool. The welding process with weaving techniques can be repeated through pool welding to get more heat in the process. A combination of the values of linear acceleration, angular acceleration, and magnetic force can be used to monitor typical signal-forming characteristics of welder hand motion.

\subsection{Recognition of Qualified (Q) and Unqualified (UQ) Welders}

Recently, developments in human activity monitoring have been increasing in various sectors. Researchers compete in undertaking significant developments to meet the industrial demand for real-time monitoring. Various types of equipment, such as cameras, PIR, and wearable motion sensors (GPS, accelerometers, gyroscopes, and magnetometers) are often applied in monitoring human activity (Dong et al., 2019; Qin et al., 2020). However, only common human activities (sleeping, standing, walking, standing, and climbing) have been carried out by the researchers. No such system has been implemented in industrial welding activities.

The present study aims to determine whether a wearable device equipped with an accelerometer, a gyroscope, and a magnetometer can be used to monitor and record wristhand motions. Each sensor has 3 DOFs in the direction of X, Y, and Z, providing a total of 9DOF motion monitoring. After the measurement data are recorded, the use of the SVM method with a supervised learning approach is explored to classify and to recognize the wrist-hand motions of Q and UQ welders. The MATLAB AI software (MATLAB, 2020) is used to process the data, to perform a data-training procedure, and to test the accuracy of the recognition.

\section{Methods}

This study used four main steps, including locating sensors, monitoring and recording hand motions during the welding experiment, preprocessing, and classifying data. The 
wearable device was attached to each welder's wrist. The activities were monitored and recorded using a MetaMotion sensor made by MbientLab Inc, USA. The device offers realtime monitoring of motions and environmental conditions. All data were stored on a smartphone and then uploaded to a cloud server. An illustration of this experimental method is shown in Figure 1.

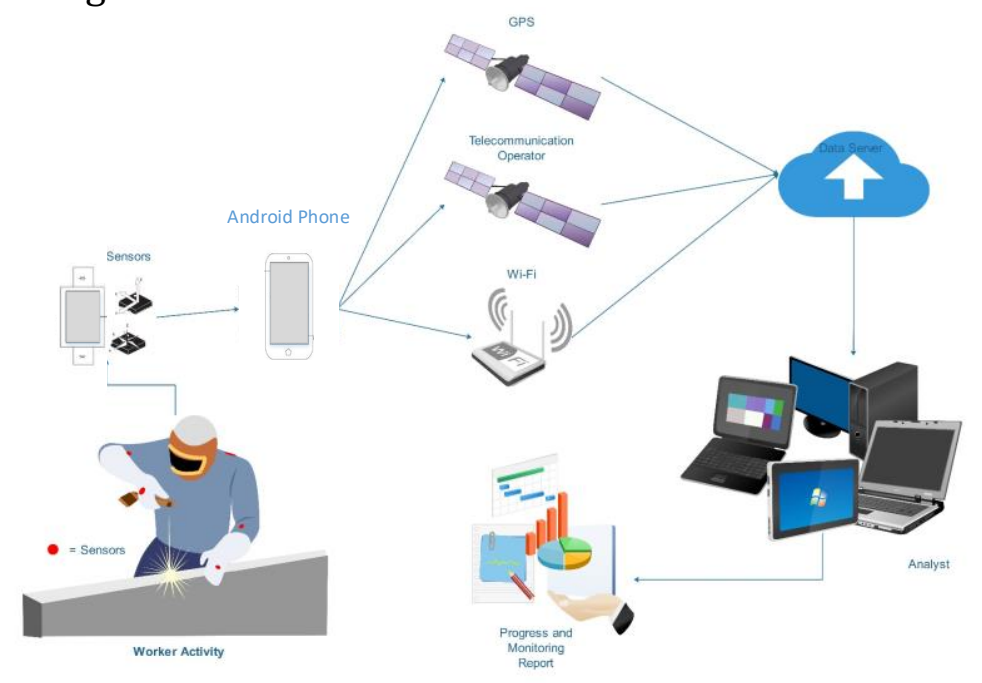

Figure 1 Illustration of monitoring and recording system for data collection using wearable sensors

Figure 2 illustrates the overall process of monitoring and recording, including the fixed parameters and the observed parameters during the experiment. Both parameters are referred to as the qualified WPS, and this study focuses on the parameters affected by the wrist-hand motions of the welders.

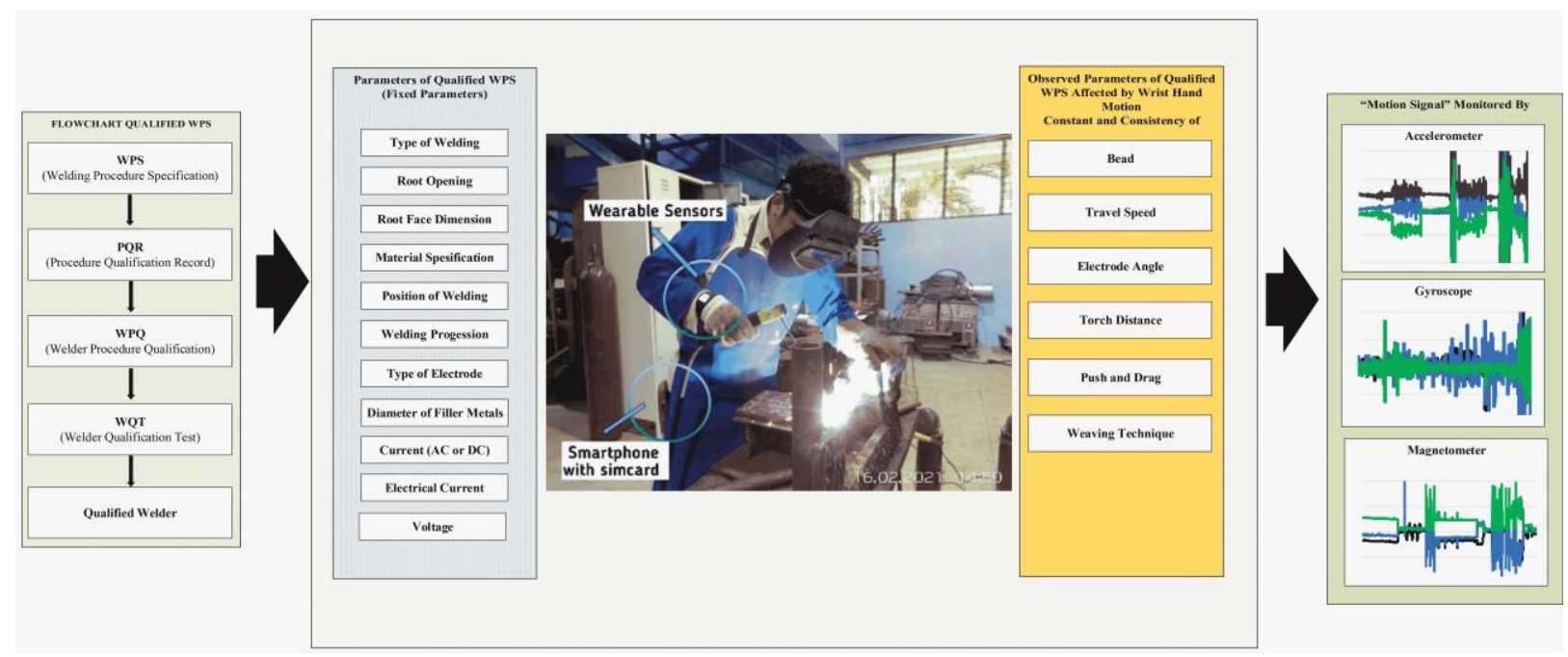

Figure 2 Flow chart research and wearable sensors for monitoring hand motions of a welder

Four base metal specimens were prepared. They were made of carbon steel material (A36), according to the WPS. The dimensions of the specimens were $150 \times 300 \times 10 \mathrm{~mm}$, with a V groove of 3-32 mm. Two Q welders and two UQ welders were assigned to perform four layers of butt joint welding in each specimen to reach the dimensions of $300 \times 300 \times 10 \mathrm{~mm}$. The welding process was performed using the ESAB Buddy ARC400i welding machine (ESAB, USA) at a 22-V DC polarity voltage and a current of 75-120 amperes. The electrodes used were E7016 LB52. The experiments were conducted in the Ship Production 
Technology and Management Laboratory, Sepuluh Nopember Institute of Technology. The results were visually inspected by a certified welding inspector. In total, 258 window samples were examined: 120 were from Q welders, and 138 were from UQ welders.

The preprocessing stage comprised data segmentation and feature extraction, executed using the MATLAB software (MATLAB, 2020). The measurement data obtained from the experiments were processed, as performed by Pribadi and Shinoda (2020). The raw data were divided into windows of 5 seconds. The measurement frequency was $25 \mathrm{~Hz}$, resulting in a total of 125 values from each sensor. Three sensors, each with three axes (x, y, and z), were used to simultaneously measure the wrist-hand motions of welders.

The extraction process was performed both in the time domain and the frequency domain analysis. The goal was to find certain characteristics, called the typical features, that represent Q and UQ welders. Several parameters were examined, including the mean, the root mean square (RMS), autocorrelation, spectral peak, and spectral power.

The classification process with the featured data is part of the identification process recommended in the machine learning process. The SVM method is one kind of classification in the machine learning technique. At the early stage, a classification in the two optimal categories, i.e. image processing and text recognition can use the SVM in linear separation (Qian et al., 2010). Currently, the SVM can be applied to perform classification processes in various categories (Hsu and Lin, 2002). One-vs-All (OvA) classification and One-vs-One (OvO) classification are the common methods used in completing multipleclass classification tasks (Alpaydin, 2014).

During the processing analysis, all window data were extracted, related to the commonly used features of time-series signal analysis, as shown in Table 1. Furthermore, a labeling process was carried out to obtain a set of training data for the next step. At the final stage, the supervised learning algorithm was used to develop the SVM Classifier model for the set of training data. The classifier model's results were then verified from the testing data set, following the k-fold cross-validation method.

\section{Results and Discussion}

\subsection{Data Acquisition}

The motion data samples from the welders were captured and acquired by the three sensors. Two examples of the results of the weldment and the corresponding results of signal graphs, including data acquisition of the $1 G$ position for $Q$ and UQ welders, are shown in Figure 3. On one hand, a $\mathrm{Q}$ welder generates relatively smooth and sound weldment results, which can be related to the acquisition signal showing a continuous and relatively constant signal graph (Areas A-F). However, Area G in the graph shows unusual welding results at the end of the welding process.

On the other hand, the welding results produced by a UQ welder show discontinuities and irregularities, which are indicated by a visual inspection of Areas A, E, and G for incomplete penetration and Areas B, C, and F for incomplete fusion, while Area D does not show any discontinuity in the welding results. The corresponding data acquisition sensor graph signal shows several typical noise patterns of the three sensors in related areas (AG) from the start to the end of the welding process performed by a UQ welder. The weldment results are significantly different from those generated by a $\mathrm{Q}$ welder.

It can be shown that the two signal graphs differ because of the varying capabilities of the two welders maintaining the stability of their hand motions during the welding process. Such capability is significant in shipbuilding, where the welder must perform the job for a long time compared with the welding specimen. The incomplete penetration defect occurs due to the increased linear acceleration of hand motions. The incomplete fusion defect 
occurs due to the increased degree acceleration of hand motions.

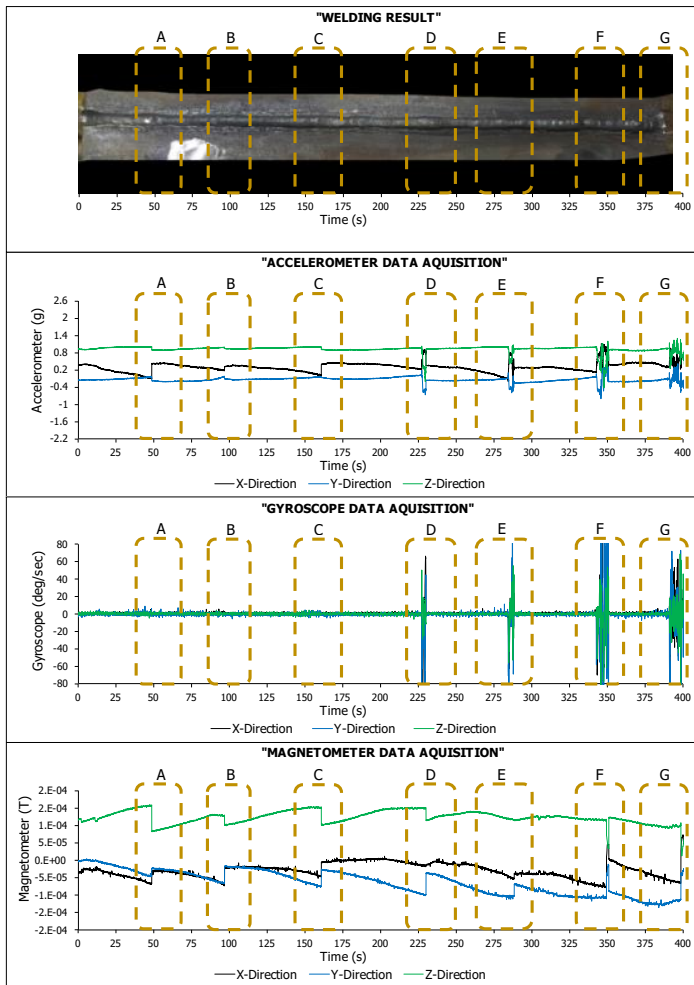

(a)

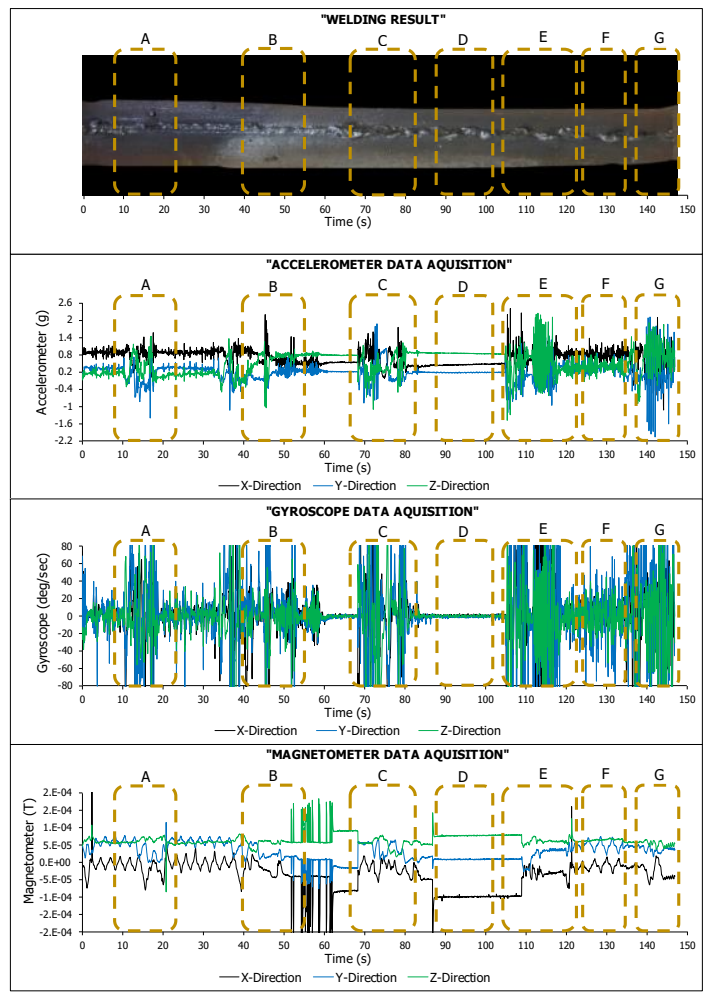

(b)

Figure 3 A comparison of $1 \mathrm{G}$ weldment visual inspection and corresponding graphs of accelerometer, gyroscope, and magnetometer: (a) qualified welder; and (b) unqualified welder

\subsection{Classification of Measurement Data}

The data set for the $1 \mathrm{G}$ welding position was used as a sample in experimenting with classifying $Q$ and UQ welders. A subset of the data was generated from the grouping process in the data window.

The properties of time and frequency from the processing of signals could be generated from the extraction of every window of data. Its properties include the mean, the RMS, autocorrelation, and power spectral features. Table 1 presents the feature extraction and functions applied and shows examples of the resulting extraction for three windows of accelerometer-x that typically distinguish between $Q$ and UQ welders. The significant difference in the extraction values between the $Q$ and the UQ welders can be seen in the average of mean, autocorrelation, and spectral power values. It is this extraction value that typically distinguishes between the welding signals from Q and UQ welders.

In the experiment, gravity exerted some influence on the resulting data. Data normalization was conducted with a high-pass filter to eliminate the gravity factor. The identification process for a Q welder and a UQ welder was provided with label identities 1 and 2, respectively, as shown in Table 2 .

The data on the extracted features were plotted as points in $n$-dimensional space (where $n$ denotes the number of features), with the value of each feature being the value of a particular coordinate. The extracted data were then classified using an SVM supervised learning algorithm. Then, the classifying process was performed by finding the hyper-plane that differentiated between the two classes UQ and Q. Such a process was executed simultaneously for the number of extracted features. 


\subsection{Welder Category Recognition Results}

The MATLAB software licensed to ITS was used to define and analyze the measured data from the sensors, received by smartphones and connected to the cloud server. The recognition of the rate of the testing data for the two welder categories (ID 1 and ID 2), using several types of multi-class SVM approaches, is shown in Table 2.

Table 1 Features used to classify Q and UQ welders and results of extraction

\begin{tabular}{|c|c|c|c|c|c|c|c|}
\hline \multirow{2}{*}{ Features } & \multirow{2}{*}{ Function } & \multicolumn{3}{|c|}{ Qualified Welder (acc x) } & \multicolumn{3}{|c|}{ Unqualified Welder (acc x) } \\
\hline & & w1 & w2 & w3 & w1 & w2 & w3 \\
\hline Mean & $\bar{w}=\frac{1}{N} \sum_{i=1}^{N} w_{i}$ & 0.3599 & 0.2800 & 0.2298 & 0.8053 & 0.8087 & 0.8127 \\
\hline $\begin{array}{l}\text { Root mean } \\
\text { square (RMS) }\end{array}$ & $\begin{array}{l}R M S(w)=\sqrt{\frac{1}{n}} \sum_{i=1}^{n} w_{i}^{2} \\
d_{P A C F}(\boldsymbol{x}, \boldsymbol{y})\end{array}$ & 0.0555 & 0.0375 & 0.0342 & 0.09501 & 0.0954 & 0.0955 \\
\hline $\begin{array}{l}\text { Auto- } \\
\text { correlation }\end{array}$ & $=\sqrt{\sum_{l=1}^{L}\left(\hat{\phi}_{l l}^{x}-\hat{\phi}_{l l}^{y}\right)^{2}}$ & 0.7701 & 0.3518 & 0.2923 & 2.2567 & 2.2736 & 2.2809 \\
\hline $\begin{array}{l}\text { Spectral } \\
\text { peaks }\end{array}$ & $\begin{array}{l}h\left(k_{\beta}\right) \\
\triangleq \frac{\left|X\left(k_{\beta}\right)\right|}{\left[\left|X\left(k_{\gamma}-\right)\right|+\left|X\left(k_{\gamma}+\right)\right|\right] /\llcorner}\end{array}$ & 0.4272 & 0.4150 & 0.4272 & 0.4211 & 0.4211 & 0.4211 \\
\hline $\begin{array}{l}\text { Spectral } \\
\text { power }\end{array}$ & $\begin{array}{l}S_{m}[n] \\
=\frac{2 K P}{f_{s} W_{s s}} \sum_{l=0}^{P-1}\left|\tilde{u}_{m l}[n]\right|^{2}\end{array}$ & 0.2478 & 0.1063 & 0.0936 & 0.7271 & 0.7278 & 0.7371 \\
\hline
\end{tabular}

Table 2 The recognition rate of testing data for Q and UQ welder categories

\begin{tabular}{cccc}
\hline ID & Welder category & Right recognition rate (\%) & Mean rate (\%) \\
\hline 1 & Qualified welder & 94.4 & 92.3 \\
2 & Unqualified welder & 90.5 & 92.3 \\
\hline
\end{tabular}

The results of the training data for the welder categories are shown as a separated scatter plot in Figure 4a, and the results of the category prediction accuracy of the testing data are figured out in the confusion matrix shown in Figure 4b. Figure 4a shows examples of the UQ and the $Q$ welder extract data, classified by the two features (TotalAccYMean and TotalAccXMean).

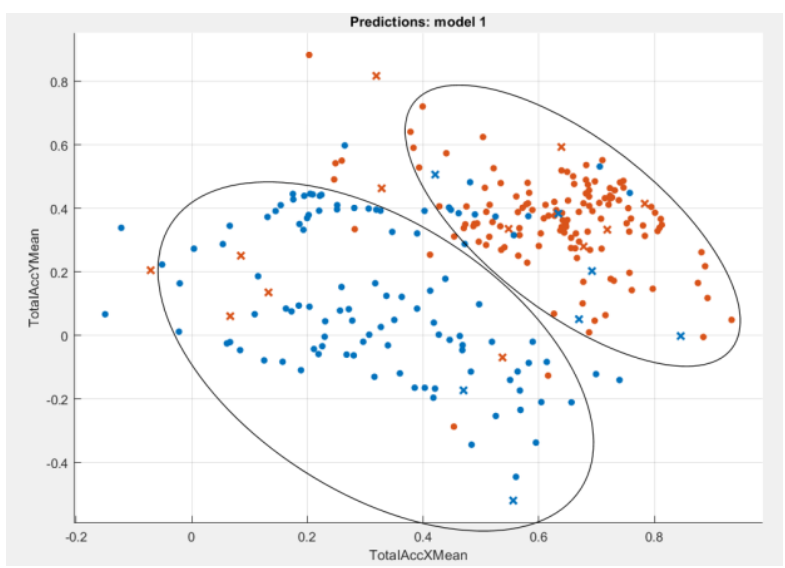

(a)

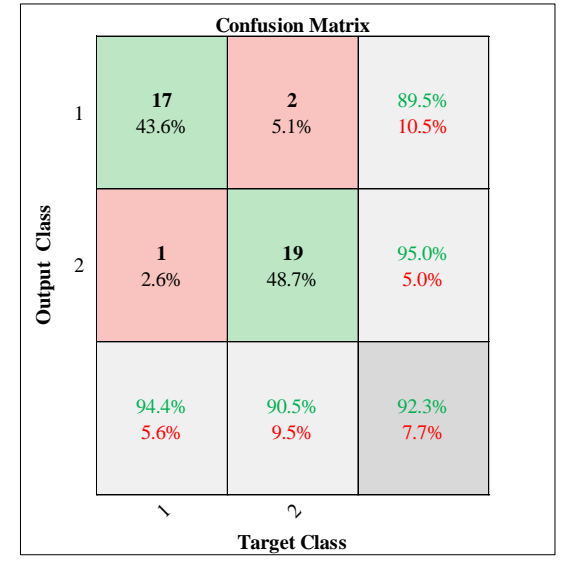

(b)

Figure 4 (a) Results of quadratic SVM training; and (b) confusion matrix of testing 
Figure $4 \mathrm{~b}$ shows the outcomes of the confusion matrix tested from 39 windows, representing $20 \%$ of the sample. The testing data consist of 19 windows from the Q welders and 20 windows from the UQ welders. The confusion matrix demonstrates the following:

(1) Seventeen windows of the qualified test data are correct (43.6\% of the 39 windows), or 17 of the 19 windows from the $Q$ welders are valid (89.5\%).

(2) Nineteen windows of the unqualified test data are correct (48.7\% of the 39 windows), or 19 of the 20 windows from the UQ welders are valid (95.0\%).

In total, 36 out of the 39 windows (92.3\%) are considered valid predictions (of the welders' qualifications).

This study attempted to assess the application of the wearable device and the SVM machine learning method to monitor and to recognize $Q$ and UQ welders. The overall results show reasonable outcomes based on the mean recognition rate. The prediction accuracy tends to increase when the number of the data measurements of Q and UQ welders is added and processed as training data.

The welders selected as samples in this study were well-defined-one Q and one UQ. The results show quite a significant distinction. One question that might be raised is whether the method would be effective if the welders' qualifications do not differ substantially (gradually). Similar inquiries could also be raised regarding other welding positions.

Additionally, it is essential to emphasize that methodological problems in the research design limit the authors' interpretations. Applications of other machine learning methods, such as the multilayer perceptron and deep learning, should also be explored.

\section{Conclusions}

This study examined the potential of utilizing real-time monitoring by means of a wearable device to differentiate between Q and UQ welders. Various data patterns can be extracted using wearable devices. A database consisting of hand motion patterns by $Q$ and UQ welders was developed as a reference for further identification of welders' qualifications. The proposed method can be considered a novel and cost-effective approach to overcoming some practical steel construction problems, including those in shipbuilding. Welding supervisors could use this method to reduce contact with welders, without compromising the quality of the results in uncertain conditions, such as during the COVID19 pandemic. This system could also be used to identify $Q$ welders who might apply improper hand motions because of fatigue or decreased focus, as well as to identify a replacement of a Q welder with a UQ one. The supervisors could monitor the performance of welders using a web-based monitoring system and respond accordingly.

\section{Acknowledgements}

The authors thank all members of PT Dok dan Perkapalan (DPS) Surabaya for the opportunity to observe the welding practices in the shipbuilding process.

\section{References}

Alpaydin, E., 2014. Introduction to Machine Learning Ethem Alpaydin. Introduction to Machine Learning, Third Edition. MIT Press. Massachusetts. pp. 32-36

Attal, F., Mohammed, S., Dedabrishvili, M., Chamroukhi, F., Oukhellou, L., Amirat, Y., 2015. Physical Human Activity Recognition using Wearable Sensors. Sensors, Volume 15(12), pp. 31314-31338

Bayat, A., Pomplun, M., Tran, D.A., 2014. A Study on Human Activity Recognition using 
Accelerometer Data from Smartphones. Procedia Computer Science. Volume 34, pp. 450457

Bortolini, M., Faccio, M., Gamberi, M., Pilati, F., 2018. Motion Analysis System (MAS) for Production and Ergonomics Assessment in the Manufacturing Processes. Computers \& Industrial Engineering, Volume 139, https://doi.org/10.1016/j.cie.2018.10.046

Bowen, J., Hinze, A., Griffiths, C., 2019. Investigating Real-Time Monitoring of Fatigue Indicators of New Zealand Forestry Workers. Accident Analysis and Prevention, Volume 126, pp. $122-141$

Dong, J., Xia, Z., Yan, W., Zhao, Q., 2019. Dynamic Gesture Recognition by Directional Pulse Coupled Neural Networks for Human-Robot Interaction in Real Time. Journal of Visual Communication and Image Representation, Volume 63, https://doi.org/10.1016/j.jvcir.2019.102583

Erasmus+, 2014. Mechanized, Orbital and Robot Welding-Mechanized Welding, pp. 1-94

Everett, T., Kell, C., 2010. Human Movement: An Introductory Text. Churchill Livingstone. Churchill Livingstone/Elsevier, New York, pp. 105-109

Godfrey, A., Conway, R., Meagher, D., ÓLaighin, G., 2008. Direct Measurement of Human Movement by Accelerometry. Medical Engineering and Physics, Volume 30(10), pp. 1364-1386

Gyllensten, I.C., 2010. Physical Activity Recognition in Daily Life using a Triaxial Accelerometer. PhD thesis, KTH Royal Institute of Technology.

Hsu, C.W., Lin, C.J., 2002. A Comparison of Methods for Multiclass Support Vector Machines. IEEE Transactions on Neural Networks, Volume 13(2), pp. 415-425

Jiang, W., Yin, Z., 2015. Human Activity Recognition using Wearable Sensors by Deep Convolutional Neural Networks. In: Proceedings of the 2015 ACM Multimedia Conference, pp. 1307-1310

Keshavarzian, A., Sharifian, S., Seyedin, S., 2019. Modified Deep Residual Network Architecture Deployed on Serverless Framework of IoT Platform based on Human Activity Recognition Application. Future Generation Computer Systems, Volume 101, pp. 14-28

Kong, L., Li, H., Yu, Y., Luo, H., Skitmore, M., Antwi-Afari, M.F., 2018. Quantifying the Physical Intensity of Construction Workers, a Mechanical Energy Approach. Advanced Engineering Informatics, Volume 38, pp. 404-419

Lee, W., Lin, K.Y., Seto, E., Migliaccio, G.C., 2017. Wearable Sensors for Monitoring On-Duty and Off-Duty Worker Physiological Status and Activities in Construction. Automation in Construction, Volume 83, pp. 341-353

MATLAB, 2020. Natick, Massachusetts: The MathWorks Inc. Mathworks: USA

Moore, P., Booth, G., 2015. 3-Welding problems and defects. P. Moore \& G. Booth, ed., The Welding Engineer's Guide to Fracture and Fatigue. Oxford: Woodhead Publishing, pp. 23-36

Pribadi, T.W., Shinoda, T., 2020. Hand Motion Recognition of Shipyard Welder using 9-DOF Inertial Measurement Unit and Multi Layer Perceptron Approach. In: IOP Conference Series: Earth and Environmental Science, Volume 557, pp. 1-15

Purnama, D., Winarto, W., Sofyan, N., Prihastomo, A., Ito, K., 2020. Microstructure and Mechanical Properties of Ah-36 Steel Weldment Welded using Magnesium Modified E6013 Electrode. International Journal of Technology, Volume 11(1), pp. 48-59

Qian, H., Mao, Y., Xiang, W., Wang, Z., 2010. Recognition of Human Activities using SVM Multi-Class Classifier. Pattern Recognition Letters, Volume 31(2), pp. 100-111

Qin, Z., Zhang, Y., Meng, S., Qin, Z., Choo, K.-K.R., 2020. Imaging and Fusing Time Series for Wearable Sensor-Based Human Activity Recognition. Information Fusion, Volume 53, 
pp. 80-87

Sunar, A., Reggi, B., Kurniawan, P., Haikal., 2019. Evaluation of the 2-Axis Movement of a 5Axis Gantry Robot for Welding Applications. International Journal of Technology, Volume 10(5), pp. 1024-1032

Valero, E., Sivanathan, A., Bosché, F., Abdel-Wahab, M., 2017. Analysis of Construction Trade Worker Body Motions using a Wearable and Wireless Motion Sensor Network. Automation in Construction, Volume 83, pp. 48-55 